\title{
Beneficios de la práctica de Mindfulness y el proceso atencional: una revisión teórica sobre su relación
}

\author{
Benefits of Mindfulness Practice and the Attentional Process: a Theoretical Review of their \\ Relationship
}

Jorge A. Guzmán-Cortés ${ }^{a}$, Hilda. C. Calvillo ${ }^{b}$, Jorge Bernal $^{c}$, Ángel F. Villalva-Sánchez $^{d}$

\begin{abstract}
:
Meditation, although it has been practiced for more than 2,500 years, has now returned to a visible force that has attracted the attention of researchers from different branches: psychology, medicine and neurosciences have turned to know the usefulness of strategies of meditation in improving the health of people. Mindfulness, a meditation defined as "a form of attention that is non-elaborative, nonjudgmental, centered in the present, in which every thought, feeling or sensation that appears in the attentional field is recognized and accepted as itis" (Bishop et al. al., 2004), has been related as a useful strategy for modifying neural functional networks and improving attention. Mindfulness meditation through its three main strategies, focused attention, open monitoring and lovingkindness, has been taken up by psychology applying it within different cognitive interventions. This document describes some of the most recent research on Mindfulness meditation and its relationship with the improvement of Attention, as well as the agreements and discrepancies that exist at present about its possible benefits, which makes the investigation of meditation and brain activity in a cutting-edge subject in the field of psychology and neurosciences
\end{abstract}

Keywords:

Mindfulness, meditation, Attention

\section{Resumen:}

La meditación, si bien ha sido practicada desde hace más de 2,500 años, en la actualidad ha retomado una visible vigencia que ha llamado la atención de investigadores de distintas ramas: la psicología, la medicina y las neurociencias se han volcado para conocer la utilidad de estrategias de meditación en la mejora de la salud de las personas. El Mindfulness, una meditación definida como "una forma de atención no elaborativa, que no juzga, centrada en el presente, en la que cada pensamiento, sentimiento o sensación que aparece en el campo atencional es reconocida y aceptada tal como es" (Bishop et al., 2004), ha sido relacionada como una estrategia útil para la modificación de redes funcionales neuronales y la mejora de atención. La meditación Mindfulness a través de sus tres principales estrategias, atención focalizada, monitoreo abierto y bondad amorosa, ha sido retomada por la psicología aplicándola dentro de diferentes intervenciones de corte cognitivo. El presente documento describe algunas de las investigaciones más recientes en torno a la meditación Mindfulness y su vinculación con la mejora de la Atención, así como los acuerdos y discrepancias que existen en la actualidad acerca de sus posibles beneficios, lo que convierte a la investigación de la meditación y la actividad cerebral en un tema de vanguardia en el campo de la psicología y las neurociencias.

\section{Palabras Clave:}

Mindfulness, meditación, atención

\section{Introducción}

La práctica de Mindfulness como estrategia de meditación, ha sido practicada desde hace 2,500 años con la finalidad de aliviar el sufrimiento humano (Pollak,
Pedulla \& Siegel, 2016). El término Mindfulness deriva de la palabra "sati" de origen pali, ha sido interpretado como conciencia, atención y recuerdo; esto último haciendo referencia al hecho de recordar que tenemos que permanecer conscientes y prestar atención, de ahí que el término Mindfulness sea traducido como conciencia plena

a Autor de correspondencia, Escuela Superior de Actopan, Universidad Autónoma del Estado de Hidalgo, E-mail jorge_guzman@uaeh.edu.mx

b Universidad Nacional Autónoma de México. Facultad de Estudios Superiores Iztacala, E-mail hilda.calvillo@hotmail.com

c Instituto de Neuropsicología y Neurociencias Cognitivas (INNCOG), E-mail jbernal@unam.mx 
(Pérez y Botella, 2006). El Mindfulness ha retomado popularidad en los últimos años entre las personas que buscan el mejoramiento de su salud. Se ha reportado que practicar este tipo de meditación está relacionado con beneficios en el estado de ánimo, con lo cual se logran mejorías en los procesos cognitivos y en la actividad cerebral.

La práctica del Mindfulness se basa principalmente en tres estrategias/habilidades que se van desarrollando (Pollak et al, 2016):

Atención focalizada o concentración: se considera un buen punto de partida para comenzar con la práctica de Mindfulness, a través de la realización de ejercicios de concentración para estabilizar la mente, básicamente, el objeto principal sobre el cual se postra la atención del individuo, puede ser cualquier cosa, desde las sensaciones que produce la respiración hasta una situación externa como el sonido del viento. Por lo regular, llegarán a la mente otros pensamientos provocando otras sensaciones; en este tipo de estrategia, si la mente comienza a divagar, se redirige la atención al primer objeto al cual se puso atención al comienzo de la meditación y en la que focalizamos los sentidos, es decir que se debe continuar con la consciencia metacognitiva, ser conscientes de lo que la mente hace.

Monitoreo abierto: una vez que la mente ha desarrollado la habilidad de permanecer concentrado en un objeto y es capaz de darse cuenta cuando se desvía la atención hacia otro objeto, resulta viable comenzar a practicar ejercicios de monitoreo abierto. A través de esta estrategia, se practica recibir con aceptación sensaciones que han sido evitadas con anterioridad, y en medida de que se aceptan, se convierten en emociones familiares en vez de intrusiones que provocan evitación.

Aceptación compasiva: esta estrategia hace uso de técnicas de autocompasión, ecuanimidad y bondad amorosa. Se pueden encontrar antecedentes de esta estrategia en la práctica de oraciones en diferentes culturas, tanto antiguas como vigentes, y han sido utilizadas con el fin de sentir apoyo en momentos de sufrimiento emocional, por lo que se ha reportado que dichos ejercicios pueden mejorar la capacidad para soportar experiencias difíciles, elevando la estabilidad emocional.

La meditación Mindfulness ha sido retomada recientemente por disciplinas de la salud como la medicina y psicología. Ha sido aplicada en el tratamiento de pacientes con diversas afectaciones, desde problemas de aprendizaje, Trastorno por Déficit de Atención e Hiperactividad (TDAH), insomnio, depresión, adicciones e incluso cáncer (Vásquez-Dextre, 2016; Chiesa \& Serretti, 2011; Shennan, Payne, \& Fenlon, 2011). Existe registro del uso de estrategias de meditación en psicoterapia desde los años 70 (Pérez y Botella, 2006), y aunque el primer trabajo sobre Mindfulness apareció en 1975, es hasta 2002 que comienza un incremento sostenido de investigaciones relacionadas a la conciencia plena, publicadas en revistas científicas (Pérez y Botella, 2006). Según Pérez y Botella (2006), del año 2002 al 2006 se registraron 398 trabajos con el término Mindfulness como título en las bases de datos Psyclnfo y Medline. Lo anterior indica que existe un interés en conocer y desarrollar teoría sobre el término, definiéndola operativamente, puntualizando sus componentes y sus mecanismos de acción, así como el desarrollo de instrumentos que permitan evaluar y comprobar la eficacia de incorporar dicha estrategia dentro de la terapia psicológica.

Es debido a la práctica atencional en la que se basa la meditación Mindfulness, que se ha despertado un creciente interés en la investigación sobre la relación entre ambas variables y en la posible mejora de la atención como consecuencia de la práctica meditativa. Históricamente, el estudio de la atención, desde la psicología, ha sido diverso con relación al paradigma desde el cuál se observe. En un primer momento, se comenzó el estudio de la atención como un mecanismo de alerta vinculado a la activación fisiológica (o su nivel de activación cerebral), para posteriormente estudiar la selectividad de los procesos atencionales, la limitación de la capacidad, así como los automatismos y el procesamiento semántico no consciente, desde una postura cognitiva (Rosselló, 1998). Ahora bien, desde una perspectiva neuropsicológica, autores como Posner y Petersen (1990) han propuesto dividir el sistema atencional en subsistemas o redes que se desempeñan de manera diferente, pero con funciones interrelacionadas: Red atencional Posterior 0 de Orientación, Red de Vigilancia o Alerta y Red Anterior o de Control Ejecutivo.

Con la finalidad de conocer las investigaciones recientes que involucran la meditación Mindfulness y las mejoras cognitivas, en específico a la atención, se realizó una búsqueda en bases de datos como ScienceDirect $y$ PubMed que arrojó resultados contenidos en revistas científicas como Springer, PLOS ONE, NatureReviews/neuroscience, Elsevier, entre otras. Se utilizaron las palabras clave "Mindfulness" y "Attention", y se seleccionaron los artículos publicados en los últimos 5 años, en inglés o español, donde se hiciera mención de ambas variables. De estos resultados se recopilaron 8 artículos que involucran Mindfulness y atención, La edad de los participantes y el tipo de investigación no fueron 
criterios de exclusión para la recopilación de artículos, por lo que dentro de los participantes se incluyen niños, jóvenes adultos y adultos.

La mejora de la atención a consecuencia de la práctica Mindfulness a través de las investigaciones revisadas muestran resultados variables, por lo que, si bien, no existe un acuerdo unánime en la relación que mantiene el Mindfulness con la mejora de la atención, sí brindan referencias que pueden ayudar al mejor entendimiento de la relación existente entre estas variables en particular y la meditación y las funciones cerebrales en general, tales como control emocional y la regulación voluntaria de la atención.

Tang, Hölzel \& Posner (2015) describen la existencia de cambios en la morfometría del cerebro relacionadas a la meditación Mindfulness, las cuales incluyen el grosor cortical, el volumen y la densidad de la sustancia gris. En dicha investigación se incluyó un grupo control formado por no meditadores y un grupo experimental conformado por meditadores que podían ser experimentados 0 novatos. En relación a la atención, reportan la mejora en tareas de monitoreo de conflicto en estudios longitudinales y transversales a través de entrenamiento de integración mente-cuerpo en sólo 5 días, practicando 20 minutos al día.

Otros investigadores (Mitchell, Zylowska \& Kollins, 2015; Carboni, Roach \& Fredrick, 2013) han realizado investigación sobre el impacto del entrenamiento de Mindfulness en adultos y niños con TDAH. Mientras que Mitchell y colaboradores encontraron resultados significativos tanto en la atención como en la hiperactividad, Carboni et al., (2013) sólo encuentran mejoría en la hiperactividad de los niños, la cual fue medida a través de cuestionarios que resolvían los padres y maestros de los sujetos de investigación.

Por su parte, Mitchell et al., (2015) realizaron estudios de caso, en los cuales se realizó entrenamiento en meditación utilizando el programa Mindful Awareness Practices MAP's para personas con TDAH, para adultos y adolescentes, quienes mostraron mejorías en autoinformes de inatención, impulsividad-hiperactividad, depresión y ansiedad a través de las evaluaciones realizadas antes y después del tratamiento.

Del mismo modo, Josefsson, Lindwall \& Broberg (2014) realizaron un estudio que evaluó autoinformes de conciencia plena y descentramiento, atención sostenida, bienestar psicológico, ansiedad, depresión y estilo de afrontamiento en población adulta trabajadora, sin experiencia previa en meditación. Los participantes se dividieron aleatoriamente en tres grupos: un primer grupo recibió intervención en terapia breve basada en Mindfulness, un segundo grupo que recibió entrenamiento en relajación y un tercer grupo en lista de espera. Se compararon los resultados de los tres grupos con el fin de conocer los efectos únicos del entrenamiento en Mindfulness. Se realizó un análisis de covarianza que mostró mejorías en autoinformes de conciencia plena y bienestar psicológico, sin embargo, no se encontraron diferencias en descentramiento, ansiedad, depresión, atención ejecutiva o estilo de afrontamiento, además de que no se encontraron diferencias en las mejoras al comparar el grupo Mindfulness y el grupo de relajación. Los investigadores atribuyen dichos resultados al hecho de que la intervención duró 4 semanas con un total de 7 sesiones.

Por otra parte, Morrison, Goolsarran, Rogers y Jha (2014) realizaron una intervención breve de entrenamiento de Mindfulness, con una duración total de 7 horas divididas en 7 semanas, realizándose 3 sesiones a la semana de 20 minutos cada una. Los participantes eran estudiantes universitarios y se buscó conocer si existían beneficios en la memoria de trabajo y la reducción de mindwandering o divagación mental. Se realizaron tareas que medían atención sostenida y distracción. Posterior al entrenamiento los resultados mostraron mejoras en la atención sostenida y los participantes informaron que se sentían más "en la tarea", decrementando puntajes de divagación. Sin embargo, la memoria de trabajo no mostró mejoría, por lo que los autores sugieren continuar la investigación respecto a la relación existente entre el Mindfulness y la disminución del mindwandering, así como sus beneficios en contextos académicos.

Resultados diferentes encontraron Mrazek, Franklin, Phillips, Baird \& Schooler (2013) quienes realizaron una intervención cuyos resultados mostraron mejoría de la memoria de trabajo, así como disminución de mindwandering por medio de entrenamiento breve de Mindfulness, concordando, en esta última parte, con el estudio anteriormente descrito, pero discrepando en los resultados relacionados a la memoria de trabajo. Trabajaron con 48 estudiantes de pregrado con los cuales formaron dos grupos de manera aleatoria, uno de ellos recibió entrenamiento en Mindfulness, y el segundo grupo fue asignado a clases de nutrición. La intervención tuvo una duración de 8 sesiones de 45 minutos cada una a lo largo de 2 semanas (cuatro reuniones a la semana). Durante las reuniones se explicaban las técnicas de meditación enfatizando estrategias de atención focalizada y se realizaba una práctica con una duración de 10 a 20 minutos, además se les extendieron indicaciones para que los participantes integraran las estrategias a sus 
actividades diarias, de tal manera que realizaran al menos 10 minutos de meditación Mindfulness al día. Los resultados mostraron mejoras en el desempeño de pruebas de razonamiento verbal y comprensión verbal, así como reducción de la ocurrencia de pensamientos distractores y el mejoramiento en puntajes de memoria de trabajo.

MacCoon, MacLean, Davidson, Saron \& Lutz (2014) se interesaron en conocer si la mejora de atención que se presenta a consecuencia del entrenamiento Mindfulness, podría suceder también con otro tipo de terapia. Dichos autores realizaron una comparación entre el entrenamiento en reducción de estrés basada en Mindfuness y un grupo control activo que realizó entrenamiento en relajación. Realizaron una intervención con una duración de 8 semanas a participantes designados aleatoriamente a uno u otro grupo. Los resultados mostraron que el grupo de entrenamiento Mindfulness obtuvo puntajes más altos, con relación al grupo control, en tareas de discriminación y vigilancia, sin embargo, con relación a la atención sostenida no se encontró diferencia. Concluyen que posiblemente 8 semanas de entrenamiento en Mindfulness no sean suficientes para lograr mejorías en la atención sostenida de los participantes, comparada con otras técnicas basadas en relajación.

Por su parte Jha, et al. (2015) realizaron una investigación con grupos de militares que habían recibido, como parte de su formación militar, entrenamiento de alta demanda atencional. Trabajaron con 3 grupos, dos de los cuales recibieron entrenamiento Mindfulness y un grupo control que no recibió dicho entrenamiento. Los dos grupos que realizaron meditación se diferenciaban en que uno de ellos, dedicó más horas prácticas de Mindfulness, mientras que el segundo grupo, dedicó más horas al aspecto teórico del Mindfulness. El entrenamiento fue diseñado a manera de terapia breve, la cual tuvo una duración de 8 horas a lo largo de 8 semanas, en las que recibían una sesión de 1 hora por semana. Ambos grupos recibieron la indicación de practicar por su cuenta 30 minutos diarios de meditación, además el grupo control fue comparado con un cuarto grupo formando por civiles, para conocer el desempeño atencional de soldados que habían recibido entrenamiento militar altamente demandante. Los resultados que encontraron indican que la estrategia del primer grupo que recibió entrenamiento práctico puede reforzar el desempeño atencional más que el grupo con entrenamiento teórico, lo que indica una mejora en el desempeño de atención por medio de la práctica Mindfulness. Finalmente, también se reportó que el grupo con entrenamiento teórico mostró un mejor desempeño atencional posterior al entrenamiento práctico en comparación al grupo civil con el que se le comparó.

\section{Conclusiones}

Se lograron identificar algunos de los avances que ha alcanzado la utilización de estrategias de meditación en el mejoramiento de los procesos cognitivos, principalmente en atención. Las modificaciones de la actividad cerebral a consecuencia de la práctica de meditación Mindfulness se encuentra actualmente en exploración, por lo que no es posible aún realizar un único juicio acerca de su eficacia y mucho menos sobre la mejor manera de aplicar la estrategia. Es por ello que futuras investigaciones podrían dirigirse en conocer la periodicidad adecuada para la práctica de meditación Mindfulness con la consecuente obtención de resultados favorables, resolviendo preguntas acerca del número de días a la semana y de horas por día que resultaría conveniente la práctica de meditación para obtener resultados a corto o mediano plazo, además de conocer por cuánto tiempo se conservan dichos beneficios una vez suspendida la intervención. Si bien se han encontrado algunas mejorías en atención y memoria en intervenciones de tan solo dos semanas (Mrazek et al., 2013), en otros no se encontraron mejorías aunen un periodo de 7 semanas (Morrison et al., 2014).

Por otro lado, existen varias estrategias de meditación dentro de la práctica Mindfulness, por lo que investigar sobre los beneficios que cada una puede ofrecer a la salud de los participantes sería de gran importancia para la mejora de la implementación de intervenciones, o bien si la estrategia no resulta una variante relevante sino la regularidad de la práctica. Otro punto interesante para investigar sería conocer la eficacia en la prevención de enfermedades o trastornos por medio de la práctica Mindfulness.

Por ahora, los avances señalan que posiblemente existirían cambios neuroanatómicos por medio del Mindfulness mejorando la orientación y el estado de alerta, por lo que resultaría interesante conocer, si derivado de estas mejoras que incluyen el grosor cortical, el volumen y la densidad de la sustancia gris (Tang, Hölzel \& Posner, 2015), se pueda prevenir o intervenir en enfermedades neurodegenerativas. Además sería importante definir si los resultados de intervenciones por este tipo de meditación presentan diferentes beneficios según las distintas etapas del desarrollo, ya que se ha encontrado que al intervenir mediante estrategias de reducción de estrés basada en Mindfulness en población con TDAH, los participantes adultos y adolescentes 
reportaron mejorías en atención y mayor control de impulsividad (Mitchell et al., 2015), mientras que una población infantil no reportó mejoras de atención, sino únicamente en la variable hiperactividad (Carboni, Roach \& Fredrick, 2013).

Si bien los beneficios que se han reportado a partir del uso de meditación Mindfulness son diversos, su aplicabilidad a diversos déficits o trastornos, tales como ansiedad, depresión, TDAH, Trastorno de estrés post-traumático, mejoramiento de la atención ejecutiva, memoria de trabajo, habilidades para el aprendizaje, autocontrol, entre otros, aún está por definirse. Falta mucho por indagar, ya que es necesario realizar más investigaciones con un mayor control metodológico en la intervención, en los instrumentos de medición y la selección de participantes, pues existe tanta heterogeneidad en los estudios que hacen difícil de conocer sus límites y alcances a nivel clínico.

Tabla 1. Comparación de resultados de investigaciones que vinculan el Mindfulness y la atención.

\begin{tabular}{|c|c|c|c|c|}
\hline Autores & $\begin{array}{l}\text { Duración de } \\
\text { intervención }\end{array}$ & Variables de intervención & Sujetos & Resultados \\
\hline $\begin{array}{l}\text { Tang, } \\
\text { Hölzel\&Posner } \\
\text { (2015) }\end{array}$ & $\begin{array}{l}\text { Terapia } \\
\text { breve y } \\
\text { terapia a } \\
\text { largo plazo } \\
\text { (NR) }\end{array}$ & $\begin{array}{l}\text { MBTS. } \\
\text { Atención sostenida. } \\
\text { Monitoreo. } \\
\text { Orientación. } \\
\text { Alerta. }\end{array}$ & $\begin{array}{l}\text { Meditadores novatos, } \\
\text { meditadores } \\
\text { experimentados y no } \\
\text { meditadores. }\end{array}$ & $\begin{array}{l}\text { Mejoras en las primeras fases de la práctica de Mindfulness, } \\
\text { en monitoreo y orientación. Mejoras en etapas posteriores } \\
\text { en el estado de alerta. }\end{array}$ \\
\hline $\begin{array}{l}\text { Carboni, } \\
\text { Roach\&Fredrick } \\
\text { (2013) }\end{array}$ & $\begin{array}{l}\text { Terapia } \\
\text { breve (NR) }\end{array}$ & $\begin{array}{l}\text { Mindfulness. } \\
\text { Hiperactividad. } \\
\text { Déficit de atención. }\end{array}$ & $\begin{array}{l}\text { Niños de } 8 \text { años estudiantes } \\
\text { de educación básica. }\end{array}$ & $\begin{array}{l}\text { Decremento de comportamiento hiperactivo. } \\
\text { No se observaron cambios en los problemas de atención. }\end{array}$ \\
\hline $\begin{array}{l}\text { Josefsson, } \\
\text { Lindwall\&Broberg } \\
\text { (2014) }\end{array}$ & $\begin{array}{l}\text { Terapia } \\
\text { breve } \\
\text { (4 semanas, } \\
7 \text { sesiones). }\end{array}$ & $\begin{array}{l}\text { Consciencia Plena. } \\
\text { Descentramiento. } \\
\text { Atención Ejecutiva. } \\
\text { Bienestar psicológico. } \\
\text { Ansiedad. } \\
\text { Depresión. } \\
\text { Estilo de afrontamiento. }\end{array}$ & Adultos. & $\begin{array}{l}\text { Se encontraron mejorías en consciencia plena y bienestar } \\
\text { psicológico. } \\
\text { No se encontraron diferencias en descentramiento, } \\
\text { ansiedad, depresión, atención ejecutiva ni estilo de } \\
\text { afrontamiento. }\end{array}$ \\
\hline $\begin{array}{l}\text { Morrison, } \\
\text { Goolsarran, Rogers } \\
\text { y Jha (2014). }\end{array}$ & $\begin{array}{l}\text { Terapia } \\
\text { breve } \\
\text { ( } 7 \text { semanas, } \\
7 \text { sesiones) }\end{array}$ & $\begin{array}{l}\text { Mindfulness } \\
\text { Mindwandering. } \\
\text { Memoria de Trabajo. }\end{array}$ & Estudiantes universitarios. & $\begin{array}{l}\text { Mejoras en la atención sostenida y decremento en puntajes } \\
\text { de divagación. } \\
\text { No se encontraron mejorías en la memoria de trabajo. }\end{array}$ \\
\hline
\end{tabular}




\begin{tabular}{|c|c|c|c|c|}
\hline $\begin{array}{l}\text { Mrazek, Franklin, } \\
\text { Phillips, } \\
\text { Baird\&Schooler } \\
\text { (2013) }\end{array}$ & $\begin{array}{l}\text { Terapia } \\
\text { breve ( } 2 \\
\text { semanas, } 8 \\
\text { sesiones) }\end{array}$ & $\begin{array}{l}\text { Mindfulness. } \\
\text { Mindwandering. } \\
\text { Atención focalizada. } \\
\text { Memoria de trabajo. }\end{array}$ & Estudiantes universitarios. & $\begin{array}{l}\text { Mejoría en memoria de trabajo. } \\
\text { Decremento de mindwandering. }\end{array}$ \\
\hline $\begin{array}{l}\text { MacCoon, } \\
\text { MacLean, } \\
\text { Davidson, } \\
\text { Saron\&Lutz, (2014) }\end{array}$ & $\begin{array}{l}\text { Terapia } \\
\text { breve } \\
\text { (8 semanas, } \\
8 \text { sesiones) }\end{array}$ & $\begin{array}{l}\text { MBSR } \\
\text { HEP } \\
\text { Mindfulness. } \\
\text { Discriminación. } \\
\text { Vigilancia. } \\
\text { Sensibilidad de la atención. }\end{array}$ & Adultos de 19 a 59 años. & $\begin{array}{l}\text { Mejorías apenas superiores en el grupo MBSR en } \\
\text { comparación al grupo control activo en tareas de } \\
\text { discriminación y vigilancia. } \\
\text { No se encontraron diferencias en relación a la sensibilidad } \\
\text { de la atención entre los grupos. }\end{array}$ \\
\hline $\begin{array}{l}\text { Jha, Morrison, } \\
\text { Dainer-Best, } \\
\text { Parker, Rostrup\& } \\
\text { Stanley (2015) }\end{array}$ & $\begin{array}{l}\text { Terapia } \\
\text { breve } \\
\text { (8 semanas, } \\
8 \text { sesiones) }\end{array}$ & $\begin{array}{l}\text { Mindfulness. } \\
\text { Entrenamiento teórico en } \\
\text { Mindfulness. } \\
\text { Entrenamiento Práctico } \\
\text { enMindfulness. } \\
\text { Atención sostenida. }\end{array}$ & Militares. & $\begin{array}{l}\text { Mejoría en el desempeño atencional en grupos que } \\
\text { recibieron MT de forma práctica. }\end{array}$ \\
\hline
\end{tabular}

Nota: NR= No reporta número de sesiones. $M T=$ MindfulnessTrainig. MBSR= Reducción de Estrés Basada en Mindfulness. TDAH= Trastorno por Déficit de Atención con Hiperactividad. HEP= HealthEnhancementProgram

\section{Referencias}

[1] Bishop, S. R., Lau, M., Shapiro, S., Carlson, L., Anderson, N., Carmody, J., Segal, Z., Abbey, S., Speca, M., Velting, D. \&Devins, G. (2004). Mindfulness: A proposedoperationalDefinition. ClinicalPsychology: Science and Practice 11(3) 230-241 doi: 10.1093/clipsy/bph077

[2] Carboni, J. A., Roach, A. T. \& Fredrick, L. D. (2013) Impact of Mindfulness Training on the Behavior of Elementary Students with Attention-Deficit/Hyperactive Disorder. Research in Human Development 10(3), 234-251

[3] Chiesa, A., Serretti, A. \&Jakobsen, J. C. (2013) Mindfulness: Topdown or bottom-up emotion regulation strategy? Clinical Psychology Review33 82-96.doi: http://dx.doi.org/10.1016/j.cpr.2012.10.006

[4] Funes, M. J. \&Lupiáñez, J. (2003) La teoría atencional de Posner: una tarea para medir las funciones atencionales de Orientación, Alerta y Control Cognitivo y la interacción entre ellas. Psicothema.15(2), 260266.

[5] Jha, A. P., Morrison, A. B., Dainer-Best, J., Parker, S., Rostrup, N. \& Stanley, E. A. (2015) Minds "At Attention": Mindfulness training curbsattentionallapses in MilitaryCohorts. PLoS ONE 10(12)doi:10.1371/journal.pone.0116889

[6] Josefsson, T., Lindwall, M. \&Broberg, A. (2014) Theeffects of a Short-termMindfulnessBasedInterventiononSel-reportedMindfulness, Decentering, ExecutiveAttention, PsychologicalHealt, and Coping Style: ExaminingUniqueMindfulnessEffects and Mediators. Springer 5 18-35.
[7] MacCoon, D. G., MacLean, K. A., Davidson, R. J., Saron, C. D. \&Lutz A. (2014) No sustainedattentiondifferences in a longitudinal randomized trial comparingMindfulnessbased stress reduction versus active control. PLoS ONE 9(6) doi: 10.1371/journal.pone.0097551

[8] Mitchell, J. T., Zylowska, L. \&Kollins, S. H. (2015)

MindfulnessMeditation Training forAttention-

Deficit/HyperactivityDisorder in Adulthood: CurrentEmpiricalSupport, TreatmentOverview, and FutureDirections. CognBehavPract. (22)2 172-191.

[9] Morrison, A. B., Goolsarran M., Rogers, S. \&Jha, A. P. (2014) Taming a wanderingattention: Short-formMindfulness training in studentcohorts. Frontiers in Human Neuroscience7doi: 10.3389/fnhum.2013.00897.

[10] Mrazek, M. D., Franklin, M. S., Phillips, D. T., Baird B. \&Schooler, J. W. (2013) Mindfulness Training ImprovesWorkingMemoryCapacity and GRE Performance WhileReducingMindWandering. PsychologicalScience 24(5) 776-781.

[11] Ostrosky-Solís, F., Gómez, P. E., Chayo-Dichy, R. \& Flores, J. C. (2004) ¿Problemas de Atención? Un programa para su estimulación y rehabilitación. México, México: American Book Store.

[12] Pérez, M. A. y Botella, L. (2006) Conciencia Plena (Mindfulness) y Psicoterapia: Concepto, Evaluacion y Aplicaciones Clínicas. Revista de Psicoterapia XVII (66/67), 79-120.

[13] Pollak, S. M., Pedulla, T. y Siegel, R. D. (2016) Sentarse Juntos. Habilidades esenciales para una psicoterapia basada en el Mindfulness. Nueva York, EUA: Desclée De Brouwer. 
[14] Posner, M.I. (1978). Chronometric explorations of mind. Hillsdale, NJ: Erlbaum.

[15] Posner, M. I. \&Petersen, S. E. (1990) Theattentionsystem of the human brain. AnnualReview of Neuroscience, 13, 25-42.

[16] Rosselló, J. (1998) Psicología de la Atención. Introducción al estudio del mecanismo atencional. Madrid, España: Ediciones Pirámide.

[17] Santiago de Torres, J., Tornay, M. F. \& Gómez, M. E. (2006) Procesos Psicológicos Básicos. Madrid, España: McGraw Hill.

[18] Shennan, C., Payne, Sh. \&Fenlon, D. (2011) Whatistheevidenceforthe use of Mindfulness-basedinterventions in cáncer care? A review. Psycho-Oncology 20 681-697 doi:10.1002/pon.1819

[19] Tang, Y., Hölzel, B. K. \&Posner, M. I. (2015) TheNeuroscience of Mindfulnessmeditation. NatureReviews/Neuroscience 16 213-225.

[20] Vásquez-Dextre, E. R. (2016) Mindfulness: Conceptos generales, psicoterapia y aplicaciones clínicas. Revista de Neuro-Psiquiatría LXXIX (1) 42-51 